\title{
Aneurisma de arteria renal y carcinoma de células claras metastásico
}

\section{Renal Artery Aneurysm and Metastatic Clear Cell Renal Carcinoma}

\author{
Sara Ruiz Oslé ${ }^{1}$ Víctor Crespo Atín ${ }^{1} \quad$ Andrea Carlevaris Fernández ${ }^{1} \quad$ Iván Olano Grasa ${ }^{1}$ \\ Marcos Acha Pérez ${ }^{1}$ \\ 1 Departamento de Urología, Hospital de Cruces, Barakaldo, Spain \\ Address for correspondence Sara Ruiz Oslé, MD, Department of \\ Urol Colomb 2019;28:25-29. \\ Urología, Hospital de Cruces, Plaza de Cruces, s/n, Barakaldo 48903, \\ Spain (e-mail: sararuizo@gmail.com).
}

\section{Resumen \\ Palabras Clave \\ - aneurisma de arteria renal \\ - displasia fibromuscular \\ - trasplante autólogo \\ - hipertensión renovascular \\ - neoplasias renales \\ - metástasis renales}

\section{Abstract}

Introducción Los aneurismas de arteria renal son poco frecuentes, la mayoría asintomáticos. Se pueden asociar a la hipertensión arterial, fibrodisplasia, arteriosclerosis sistémica y aneurismas en otras localizaciones. Son más frecuentes en mujeres. El cáncer renal representa el 2\%-3\% de todos los tumores con una incidencia creciente en las últimas décadas. Predomina en los varones y es conocida su gran capacidad para metastatizar. Se presenta un caso clínico en el que de forma poco habitual, coexisten ambas entidades.

Caso Clínico Mujer de 52 años con antecedente de autotrasplante de riñón derecho tras cirugía de aneurisma de arteria renal. Durante el seguimiento, se objetiva masa renal en dicha unidad que precisa cirugía con resultado de carcinoma de células claras. Cuatro años más tarde progresa con metástasis a nivel de suprarrenal izquierda realizándose adrenalectomía izquierda laparoscópica.

Discusión La indicación de tratamiento de los aneurismas de arteria renal radica en evitar sus complicaciones, principalmente la rotura por su capacidad letal. Entre las técnicas aún tiene su papel la cirugía exvivo y el autotrasplante. El tratamiento del cáncer renal se basa en conseguir la radicalidad en estadios localizados; por otro lado, en enfermedad avanzada, la cirugía puede estar indicada en pacientes seleccionados. En ese caso, el seguimiento del aneurisma de arteria renal permite realizar un diagnóstico y tratamiento temprano del cáncer.

Introduction Renal artery aneurysms are rare and most of them are asymptomatic. They could be linked to arterial hypertension, fibromuscular dysplasia, systemic arteriosclerosis and other visceral aneurysms. They appear more frequently in women. Renal cancer represents 2-3\% among all the malignancies. In the last years, the incidence is increasing and it is slightly more common in men. It is Known its widely capacity to develop metastasis. We report the rare coexistence of both entities in the same patient. received

December 7, 2017

accepted

February 19, 2018

published online

May 15, 2018
DOI https://doi.org/

$10.1055 / \mathrm{s}-0038-1645852$. ISSN 0120-789X.
Copyright $($ C 2019, Sociedad Colombiana License terms de Urología. Publicado por Thieme Revinter Publicações Ltda., Rio de Janeiro, Brazil. Todos los derechos reservados.

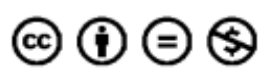




\author{
Keywords \\ - renal artery aneurysm \\ - fibromuscular \\ dysplasia \\ - transplantation \\ - autologous \\ - hypertension \\ - renovascular \\ - kidney neoplasms
}

Clinical Case Fifty-two year-old-woman, right kidney auto transplantation operated because of renal artery aneurysm surgery. In a scheduled examination, she presents a mass in that kidney, which turns out to be a clear cell carcinoma. Four years later, a left adrenal metastasis is detected and a laparoscopic adrenalectomy is performed.

Discussion Aneurysms treatment has the purpose of avoid its complications, mainly its rupture because it can cause death. Among the several techniques described, exvivo surgery and auto transplantation is one of them. Renal cancer treatment aims to be radical in localized stages. On the other hand, in some selected advanced renal cancer patients, surgery has an indication too. In the present case, the renal aneurism followup allow us to perform an early diagnosis and treatment of the neoplasm.

\section{Introducción}

Los aneurismas de arteria renal (AAR), son poco frecuentes y la mayoría asintomáticos, pero cada vez más diagnosticados debido al uso creciente de las técnicas de imagen. La etiología suele estar en relación con la displasia fibromuscular (DFM), enfermedades del colágeno, arteriosclerosis o traumatismos previos, entre otros. ${ }^{1}$ En algunas series se ha visto que predominan en la mujer por su asociación con la DFM. ${ }^{2}$ Algunas de las indicaciones para el tratamiento activo son el tamaño del aneurisma, la hipertensión arterial mal controlada y la mujer en edad fértil. Para ello, existen diferentes técnicas descritas. ${ }^{1}$

El cáncer renal (CR) representa el 2\%-3\% de todos los tumores. La incidencia se ha incrementado en las últimas décadas, predominando en varones con una ratio 1.5/1 entre los 60-70 años. Algunos de los factores etiológicos conocidos son el tabaco, obesidad, hipertensión arterial y factores genéticos. Se estima que un tercio de esos tumores desarrollan metástasis a lo largo de su evolución. ${ }^{3-5} \mathrm{El}$ tratamiento se basa en la cirugía y en terapias sistémicas dirigidas.

Se presenta un caso en el que de forma poco habitual, coexisten ambas entidades. El seguimiento periódico por imagen del aneurisma permite realizar un diagnóstico de la lesión renal en estadios iniciales.

\section{Caso Clínico}

Mujer de 52 años sin alergias medicamentosas conocidas, exfumadora de 10 paquetes/año, sin hipertensión conocida, no tomadora de anovulatorios orales ni otros tratamientos de forma habitual.

En una ecografía realizada en otro centro por dolor lumbar, se objetiva incidentalmente un aneurisma de arteria renal derecha (ARD). La arteriografía confirma dicho hallazgo de $24 \mathrm{~mm}$, en su parte distal y DFM afectando a ambas arterias renales. Acude a nuestra institución para segunda opinión, se detecta tensión arterial elevada. Inicia tratamiento con antagonistas de los receptores de angiotensina II (ARA II), y se propone reparación quirúrgica con nefrectomía, cirugía en frío y autotrasplante renal (ATR).
Se practica resección de aneurisma exvivo y reimplante renal en arteria iliaca externa derecha (AIED) realizando un bypass ilio-renal con safena interna invertido. Siete meses después se coloca endoprótesis por pseudoaneurisma en AIED.

Tras 9 años, en tomografía axial computarizada (TAC), se objetiva masa renal en cara anterior del polo inferior del autoinjerto, sugestivo de malignidad, además de aneurisma de arteria renal izquierda de $13 \mathrm{~mm}$, sin cambios respecto a la primera arteriografía ( - Fig. $\mathbf{1}$ ). Se realiza trasplantectomía por apertura incidental de tumor al intentar cirugía conservadora de nefronas. La anatomía patológica informa de carcinoma de células claras, grado 2 de Fuhrman de $3,7 \mathrm{~cm}$. Evoluciona favorablemente hasta que en TAC control, 3 años después de trasplantectomía, se detecta un nódulo suprarrenal izquierdo de $12 \mathrm{~mm}$ (-Fig. 2). Se realiza suprarrenalectomía izquierda laparoscópica sin incidencias, con resultado de metástasis del primario renal, de $1,2 \mathrm{~cm}$.

Un año después la paciente no presenta datos de recidiva a nivel local ni a distancia.

\section{Discusión}

Los AAR son dilataciones segmentarias provocadas por la debilidad del tejido elástico o el deterioro de la capa media arterial. ${ }^{5}$ Tienen una incidencia estimada de $0,09 \%,{ }^{6}$ con un incremento en su detección en los últimos años por el mayor uso de las técnicas de imagen. Se estima que representan el $22 \%$ de todos los aneurismas viscerales. ${ }^{7}$

Se clasifican según E.F. Poutasse, atendiendo a sus características morfológicas en saculares (70-90\%), ${ }^{8}$ fusiformes, disecadores y microaneurismas intrarrenales. ${ }^{9}$

Habitualmente son asintomáticos, pero entre un 55 y un $75 \%$ de esos pacientes cursan con hipertensión arterial secundaria debida a la estenosis renal segmentaria o a la compresión extrínseca. ${ }^{7}$ Otros síntomas pueden ser el dolor en flanco $(15 \%)$ o la hematuria (30\%). ${ }^{1}$

Las indicaciones para tratamiento activo más comúnmente aceptadas incluyen la ruptura y disección, hipertensión arterial mal controlada, aquellos que produzcan síntomas refractarios, tromboembolismos renales, mujeres en edad fértil y tamaño mayor de $2 \mathrm{~cm}$ o crecimiento en controles sucesivos. ${ }^{10}$ Otros autores 


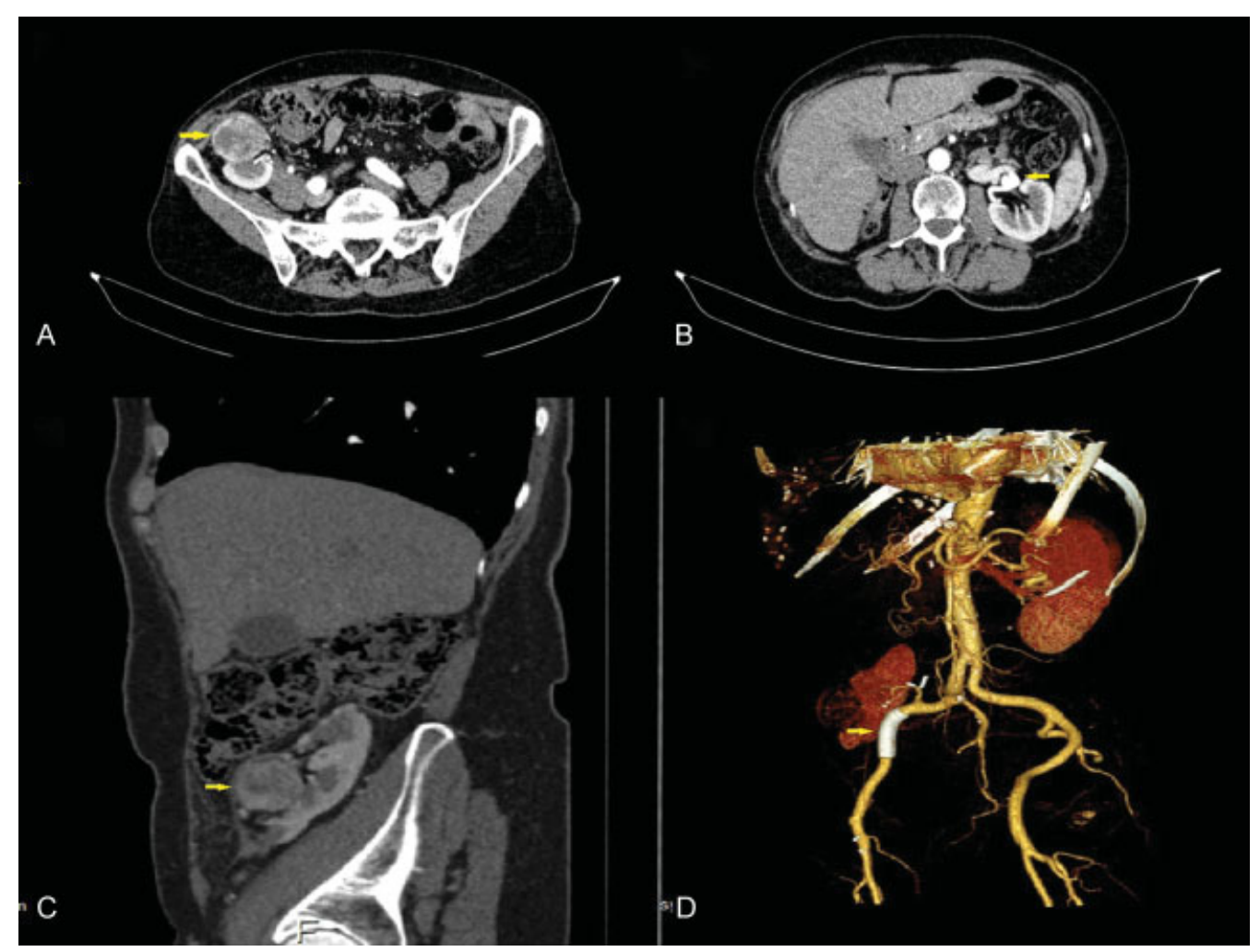

Fig. 1 Tomografía computarizada. (A y C) Corte axial y sagital: tumoración en cara anterior de polo inferior de injerto de $4 \mathrm{~cm}$ de diámetro máximo sugestivo de malignidad (flecha). (B) Corte axial: aneurisma de arteria renal izquierda de $13 \mathrm{~mm}$ (flecha). (D) Reconstrucción vascular 3D: Prótesis de AIED (flecha).

defienden la reparación independientemente de cumplir esos criterios para evitar las potenciales complicaciones. Entre ellas pueden aparecer los infartos renales, el desarrollo de hipertensión renovascular mantenida o la ruptura, que es rara (3\%) pero potencialmente letal. Durante el embarazo esa ruptura puede ser catastrófica con una mortalidad materna del $50 \%$ y fetal del $78 \%{ }^{1,6}$

Las opciones de tratamiento, además de la observación, incluyen técnicas endovasculares (embolización selectiva con coils o colocación de prótesis endovasculares) o la reparación quirúrgica, clásicamente por vía abierta. ${ }^{11,12} \mathrm{El}$ ATR, en la actualidad tiene indicaciones excepcionales:

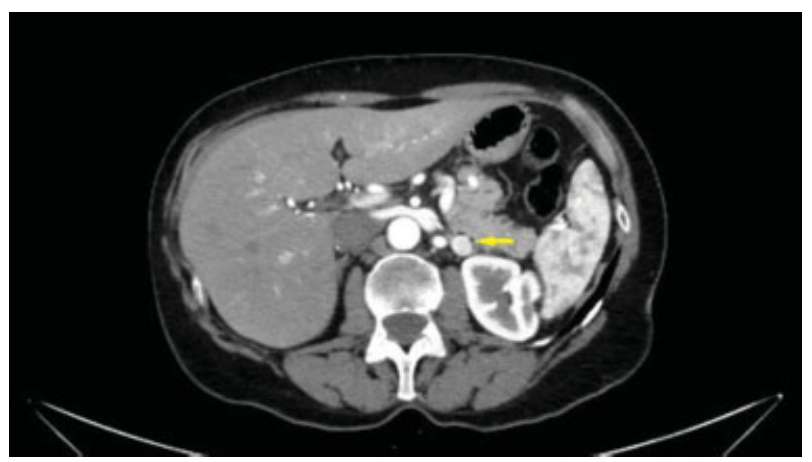

Fig. 2 Nódulo suprarrenal izquierdo de nueva aparición de $12 \mathrm{~mm}$ sospechoso de metástasis (flecha). reconstrucción exvivo de patología compleja del pedículo renal, lesiones ureterales extensas, y la cirugía conservadora del $C R$ en pacientes monorrenos o insuficientes renales con tumores que precisan cirugía de banco. De forma individualizada, se puede plantear en otros casos por permitir la reconstrucción vascular extracorpórea y la conservación de unidades renales. ${ }^{13}$ En 2001, Gill y col, publican el primer caso de reparación de AAR vía laparoscópica, ${ }^{8}$ técnica compleja y aún no muy extendida en cirugía renovascular. Gulianotti y col, en 2010, presentan una serie de 5 casos de AAR reparados vía laparoscópica asistida por robot, lo cual permite superar las dificultades técnicas de la laparoscopia convencional, así como disminuir el tiempo de isquemia. ${ }^{11}$

En nuestro caso, se indicó la cirugía por tratarse de un aneurisma mayor de $2 \mathrm{~cm}$, con importante DFM de la ARD. Tras discutir las opciones se optó por nefrectomía con conservación de la glándula adrenal, cirugía exvivo del aneurisma y posterior ATR; además se recomendó la terapia con ARA II para evitar la progresión de la DFM y control de tensión arterial, así como vigilancia de las lesiones menores de la otra unidad renal.

El CR es cada vez más frecuentemente diagnosticado de forma incidental, lo que conlleva a detectarlo con menores tamaños y en estadios asintomáticos. La triada clásica de dolor en el flanco, hematuria y masa palpable sólo aparece en el $6 \%-10 \%$. El tratamiento actual recomendado en el CR localizado es la cirugía resectiva. En estadios T1a y T1b se 
prefiere la cirugía conservadora de nefronas (CCN) siempre que sea técnicamente posible. No se recomienda la adrenalectomía ipsilateral ni la linfadenectomía si no existe evidencia de invasión de esas entidades. ${ }^{5}$ Como alternativas a la cirugía, se puede plantear la vigilancia o terapias ablativas en pacientes de alto riesgo no candidatos a tratamientos agresivos. ${ }^{3-5}$ Los sitios más comunes de desarrollo de metástasis son pulmón, hueso, hígado, cerebro, glándula adrenal y ganglios linfáticos. Entre los predictores pronósticos, destaca el grado nuclear de Fuhrman y el tipo histológico del tumor. ${ }^{5,14}$

Las opciones terapéuticas actuales en los tumores en injertos renales incluyen la $\mathrm{CCN}$ en sus diferentes modalidades en estadios $\mathrm{T} 1$, siendo la trasplantectomía de elección en T2. La mayoría de los autores abogan por una cirugía vía abierta, pues no se debe olvidar la fibrosis renal y perirrenal que existirá por el procedimiento previo. Las técnicas ablativas como la radiofrecuencia y crioablación son una alternativa en pacientes muy seleccionados. ${ }^{15}$

En nuestra paciente se indicó una nefrectomía parcial al tratarse de un tumor estadioT1a, técnicamente fue imposible por la fibrosis perirrenal y renal existente, que condicionó la apertura incidental de la masa, violándose uno de los principios básicos de la cirugía oncológica (márgenes anatómicos del tumor). ${ }^{16}$ Finalmente, se realizó trasplantectomía, manteniendo Creatinina en torno a $0.80 \mathrm{mg} / \mathrm{dl}$ y sin evidencia de enfermedad tumoral en el seguimiento salvo la mencionada.

A pesar del pobre pronóstico del CR metastásico, existen nuevas terapias, además de tratamientos sistémicos dirigidos (inmunoterapia e inhibidores de la angiogénesis). La cirugía tiene su indicación en este contexto, mediante nefrectomías citorreductoras y metastasectomías ${ }^{14}$ en el caso de pacientes seleccionados con metástasis únicas, resecables o para control sintomático. ${ }^{3-5}$

En el presente caso, se indicó adrenalectomía radical izquierda al tratarse de una lesión única, circunscrita de pequeño tamaño. La adrenalectomía parcial no fue considerada por tratarse de una lesión unilateral con la glándula contralateral preservada. ${ }^{17}$

Las pruebas de imagen en el seguimiento del paciente son clave para llevar a cabo el diagnóstico precoz de patología sin relación causal. El TAC de control tras la cirugía de AAR posibilita la detección incidental de la neoformación renal en estadio T1a con indicación de CCN, que no se puede culminar por dificultades técnicas. Ulteriores controles radiológicos revelaron la metástasis suprarrenal única susceptible de resección completa.

\section{Conclusión}

El aumento del uso de las pruebas de imagen que ha generado la medicina moderna, condiciona un incremento de diagnósticos incidentales, tanto de AAR como CR, sobre todo en estadios precoces.
El perfeccionamiento de las técnicas quirúrgicas y la evolución de la cirugía mínimamente invasiva, así como de la endocirugía, pone en manos del urólogo un amplio rango de opciones terapéuticas. El objetivo es individualizar el tratamiento teniendo en cuenta el perfil del paciente (riesgos, beneficios, sintomatología, pronóstico etc.).

\section{Responsabilidades Éticas}

Protección de personas y animales. Los autores declaran que para esta investigación no se han realizado experimentos en seres humanos ni en animales.

Confidencialidad de los datos. Los autores declaran que han seguido los protocolos de su centro de trabajo sobre la publicación de datos de pacientes.

Derecho a la privacidad y consentimiento informado. Los autores han obtenido el consentimiento informado de los pacientes y/o sujetos referidos en el artículo. Este documento obra en poder del autor de correspondencia.

\section{Bibliografía}

1 Blanco Díez A, Armas Molina J, Alvarado Rodríguez A, Alcaraz Asensio A, Artíles Hernández J, Chesa Ponce N. Aneurisma de arteria renal. Nefrectomía laparoscópica, reconstrucción en banco y autotrasplante. Actas Urol Esp 2008;32(07):763766

2 Henke PK, Cardneau JD, Welling TH III, et al. Renal artery aneurysms: a 35-year clinical experience with 252 aneurysms in 168 patients. Ann Surg 2001;234(04):454-462, discussion 462-463

3 Ljungberg B, Albiges L, Bensalah K, et al. Renal Cell Carcinoma Guidelines. European Association of Urology. 2017.

4 Ruiz-Oslé S, Prol C, Lardíes R, Gaafar A, Barbier L, Arruza A. Renal Cell Carcinoma metastases in the maxillofacial area: Case series. Arch Esp Urol 2017;70(08):732-735

5 Quiroga Matamoros W, Fernandez F, Citarella Otero D, Rangel J, Estrada Guerrero A, Darío Patiño I. Guía de manejo del carcinoma de células renales. Urol Colomb. 2016;25:169-189

6 Klausner JQ, Harlander-Locke MP, Plotnik AN, Lehrman E, DeRubertis BG, Lawrence PF. Current treatment of renal artery aneurysms may be too aggressive. J Vasc Surg 2014;59(05): 1356-1361

7 Menéndez SA, Martín SM, Fernández SP, Fajardo JA, González JR, del Busto EF. Calcified renal artery aneurism embolization in a solitary kidney. Arch Esp Urol 2011;64(07):629-631

8 Gill IS, Murphy DP, Hsu THS, Fergany A, El Fettouh H, Meraney AM. Laparoscopic repair of renal artery aneurysm. J Urol 2001;166 (01):202-205

9 Poutasse EF. Renal artery aneurysms. J Urol 1975;113(04): 443-449

10 Chen HY, Lin CC, Huang PF, et al. Surgical repair of a complex renal artery aneurysm through bench surgery and autotransplantation. Formos J Surg. 2016;49(06):233-237

11 Giulianotti PC, Bianco FM, Addeo P, Lombardi A, Coratti A, Sbrana F. Robot-assisted laparoscopic repair of renal artery aneurysms. J Vasc Surg 2010;51(04):842-849

12 Hislop SJ, Patel SA, Abt PL, Singh MJ, Illig KA. Therapy of renal artery aneurysms in New York State: outcomes of patients undergoing open and endovascular repair. Ann Vasc Surg 2009; 23(02):194-200 
13 López-Fando Lavalle L, Burgos Revilla J, Sáenz Medina J, et al. Renal autotransplantation: a valid option in the resolution of complex cases. Arch Esp Urol 2007;60(03):255-265

14 Chandrasekar T, Klaassen Z, Goldberg H, Kulkarni GS, Hamilton RJ, Fleshner NE. Metastatic renal cell carcinoma: Patterns and predictors of metastases-A contemporary population-based series. Urol Oncol 2017;35(11):661.e7-661.e14
15 Ramos E, Domínguez M, Portillo JA, et al. Therapeutic options for renal graft tumors. Arch Esp Urol 2013;66(01):180-185

16 Correa JC, Figueroa JD, Castaño R, Madrid J, Calle M, Sanabria A. Principios de cirugía oncológica. Rev Colomb Cir. 2016; 31:185-196

17 Fariña Pérez LA. Laparoscopic adrenalectomy. Actas Urol Esp 2006;30(05):510-512 